\title{
openheart Postprandial insulin assay as the earliest biomarker for diagnosing pre- diabetes, type 2 diabetes and increased cardiovascular risk
}

\author{
James J DiNicolantonio, ${ }^{1}$ Jaikrit Bhutani, ${ }^{2}$ James H OKeefe, ${ }^{1}$ Catherine Crofts $^{3}$
}

\section{To cite:}

DiNicolantonio JJ, Bhutani J, OKeefe JH, et al. Postprandial insulin assay as the earliest biomarker for diagnosing pre-diabetes, type 2 diabetes and increased cardiovascular risk. Open Heart 2017;4:e000656. doi:10.1136/ openhrt-2017-000656

Accepted 7 November 2017

\section{CrossMark}

${ }^{1}$ Preventive Cardiology, Saint Luke's Mid America Heart Institute, Kansas, Missouri, USA ${ }^{2}$ Internal Medicine, Pt BD Sharma Post Graduate Institute of Medical Sciences, Rohtak, Haryana, India ${ }^{3}$ Human Potential Centre, Auckland University of Technology, Auckland, New Zealand

Correspondence to Dr James J DiNicolantonio; jjdinicol@gmail.com

\section{INTRODUCTION}

Currently, approximately one in eleven US adults have diabetes, and more than one in three are pre-diabetic. ${ }^{1}$ Additionally, it is estimated that approximately two in five US adults will develop diabetes, with most of these cases being type 2 diabetes (T2D). ${ }^{2}$ According to data (2011-2012) recently published by Menke et al, ${ }^{3} 14.3 \%$ of US adults (over 20 years of age) were diabetic $(9.1 \%$ were diagnosed as being diabetic, $5.2 \%$ having undiagnosed diabetes) and 38\% were pre-diabetic, totalling $52.3 \%$ of US adults having pre-diabetes or diabetes. The burden of undiagnosed diabetes seems to have increased in parallel to the above estimates. Among people with diabetes, more than one-third went undiagnosed overall $(36.4 \%$ (95\% CI $30.5 \%$ to $42.7 \%)$ ). Additionally, this burden was even higher among non-Hispanic Asian participants $(50.9 \% ; \mathrm{P}=0.004)$ and Hispanic participants $(49.0 \% ; \mathrm{P}=0.02) .{ }^{3}$ In view of this, it can be concluded that using fasting glucose, oral glucose tolerance test (OGTT) or Alc may not be the most effective early screening tool for T2D. Thus, incorporating fasting insulin and especially insulin assay after an OGTT as enhanced screening methods may help to increase the ability to detect diabetes and pre-diabetes, allowing earlier intervention to prevent diabetic complications.

\section{FASTING AND POSTPRANDIAL INSULIN FOR EARLY DIAGNOSIS OF T2D \\ Kraft patterns}

Hyperinsulinaemia is thought to be aetiological in hypertension, obesity, atherosclerosis, microvascular disease, neurodegenerative disorders, idiopathic peripheral neuropathy and certain cancers, ${ }^{45}$ and is associated with idiopathic tinnitus, vertigo and hearing loss. ${ }^{6}$ A key research paper published in 1975 by Dr
Joseph R Kraft comprised 3650 patients who were randomly referred for glucose tolerance testing. ${ }^{7}$ Dr Kraft described five different insulin patterns in response to a $100 \mathrm{~g}$ glucose load administered over $3-5$ hours with insulin levels assessed at baseline, 30, 60, 120, and at minimum, $180 \mathrm{~min}$. Further 240 and $300 \mathrm{~min}$ insulin levels were also considered in certain instances. Pattern I was labelled as the normal insulin tolerance, where the fasting insulin levels were between 0 and 30 microunits, with peak insulin production demonstrated on 30 or $60 \mathrm{~min}$ levels, followed by return to fasting range at 120 or $180 \mathrm{~min}$, and stabilised on fasting levels beyond $180 \mathrm{~min}$. Pattern II accounted for those with normal insulin peak but delayed return to baseline fasting levels, and pattern III consisted of subjects with delayed insulin peak appearing later than $60 \mathrm{~min}$. Pattern IV was labelled in cases who had baseline fasting levels more than 50 microunits. The last pattern, pattern $\mathrm{V}$, had patients with insulin levels of less than 30 microunits at all times. ${ }^{7}$

These insulin/glucose tolerance tests were then compared with standard glucose tolerance tests. A score of 0 Wilkerson point was labelled as normal, $1 / 2,1$ and $1 \frac{1}{2}$ as equivocal, and 2 or 3 as diagnostic for diabetes. In this research by Kraft, $47 \%$ had normal glucose tolerance, $34 \%$ had equivocal results and $19 \%$ were positive for diabetes. The assessment of insulin levels was then performed. Pattern I was classified as normal, patterns II, III and IV as borderline or diagnostic for diabetes, and pattern $\mathrm{V}$ as low insulin. This revealed that in the normal glucose tolerance group $2.5 \%$ had low insulin, $14 \%$ were borderline diabetic and $50 \%$ had diabetic insulin levels, with only $33 \%$ having a normal insulin response to an OGTT. In the equivocal glucose tolerance group, $10 \%$ had normal 
insulin levels, while $90 \%$ had diabetic patterns, and in those with glucose tolerance test positive for diabetes, $100 \%$ of the subjects had diabetic insulin patterns. This includes 62 cases who had pattern $\mathrm{V}$ (low insulin) and a clear hyperglycaemic response consistent with type 1 diabetes. Kraft's research indicated that $75 \%$ of those subjects with normal or equivocal glucose tolerance tests $(\mathrm{n}=2940)$ had borderline or abnormal insulin response patterns (patterns II, III and IV) and likely had 'diabetes in-situ' or 'occult diabetes'.

Another similar study by Dr Kraft produced identical conclusions and also compared two different methods of insulin estimation (RIA: radioimmunoassay and MEIA: microparticle enzyme immunoassay). Using the RIA $87.8 \%(\mathrm{n}=303)$ and with MEIA $79.1 \%(\mathrm{n}=273)$ of subjects with a normal or equivocal glucose tolerance test (Wilkerson score $0,1 / 2,1$ or $\left.1 \frac{1 / 2}{2}\right)(n=345)$ were found to have abnormal insulin response patterns (pattern II, III, IV). Those with overt diabetes on glucose tolerance test (Wilkerson score 2 or 3 ) inevitably had abnormal or reduced insulin response patterns. ${ }^{8}$

The flaw in Kraft's original research is that it did not account for a number of different insulin patterns, including those who had a fasting level between 30 and 50 microunits. Working with Dr Kraft, Crofts and colleagues ${ }^{9}$ redefined Kraft's original patterns to ensure all tests could be categorised.Patterns I, IV and V remained the same, but patterns II and III were expanded to include those with a fasting insulin between 31 and 49 microunits (inclusive). Crofts $e t$ al then reanalysed the data set using WHO glucose disorder definitions. Of the 3428 people with either impaired glucose tolerance or diabetes, the vast majority (93\%) also had a hyperinsulinaemic pattern. Hyperinsulinaemia also affected approximately $75 \%$ of the people with normal glucose tolerance $(n=4030)$.

\section{Hayashi patterns}

More recent prospective research by Hayashi et $a l^{10}$ further confirmed the above findings and showed that insulin concentrations during glucose tolerance test strongly predict the development of T2D. They followed 400 non-diabetic Japanese Americans for 10-11 years to assess incidence of T2D. Each participant underwent insulin estimation at baseline, 30, 60 and 120 min intervals during a 2-hour $75 \mathrm{~g}$ OGTT. The insulin response patterns were classified into five types, distinct from Kraft's. Pattern 1 included initial insulin peak within $30 \mathrm{~min}$, and higher insulin level at $60 \mathrm{~min}$ than at $120 \mathrm{~min}$; pattern 2, with similar initial peak, but 1-hour level was less than or equal to level at 2 hours; pattern 3, initial peak within 1 hour; pattern 4 , with initial peak within first 2 hours, and a lower level at $30 \mathrm{~min}$ than at $60 \mathrm{~min}$; and pattern 5, with initial peak within 2 hours, and equal or higher level at $30 \mathrm{~min}$ as compared with $60 \mathrm{~min}$. After the follow-up 86 cases developed T2D. Patterns 1, 2, 3, 4 and 5 demonstrated a cumulative incidence of $3.2 \%, 9.8 \%$, $15.4 \%, 47.8 \%$ and $37.5 \%$, respectively. The multiple-adjusted ORs for developing T2D were 12.55 (95\% CI 4.79 to 32.89) for pattern 4, and 8.34 (95\% CI 2.38 to 29.27) for pattern 5, as opposed to patterns 1 and 2. Also, this risk was independent of insulin secretion and sensitivity.

\section{FASTING INSULIN RESPONSE ASSESSMENTS}

Multiple-sampled glucose tolerance tests required to produce the Kraft and Hayashi patterns are resource-intensive. This may explain the popularity of fasting measures such as homeostasis modal assessment (HOMA). HOMA was originally developed to assess insulin resistance (HOMA-IR), pancreatic beta-cell function (HOMA- $\beta$ ) and insulin sensitivity (HOMA-S) from fasting plasma insulin and glucose concentrations. ${ }^{11}$ This is based on the relationship between basal insulin and glucose concentrations reflecting hepatic glucose output and insulin secretion. Normal pancreatic beta-cell function or insulin sensitivity is considered $100 \%$ and normal insulin resistance is 1.0. HOMA variants can be determined either by using the calculations shown in figure 1 or by using HOMA 2 from the online calculator (https:/ / www.dtu.ox.ac.uk/homacalculator/). HOMA2 uses model-derived estimates, which better accounts for variations in glucose resistance and when plasma glucose concentrations are above $10 \mathrm{mmol} / \mathrm{L}^{12}$ However, there are numerous flaws to using measures based on fasting insulin as discussed below.

\section{Flaws in using fasting insulin resistance models}

There are several flaws with using insulin resistance models that are based on fasting insulin and glucose concentrations, including the physiology of insulin secretion and basal levels, the use of insulin resistance as the disease state of concern, and implementation and interpretation of the test itself.

\section{Insulin physiology}

Plasma insulin concentrations are labile due to the physiology of insulin release from the pancreas. Insulin is released in a pulsatile manner leading to oscillatory concentrations in the blood. These oscillations have a slow ultradian periodicity $(\sim 140 \mathrm{~min})$, which is modulated by

$$
\text { HOMA-IR }=\frac{\text { Glucose } x \text { Insulin }}{22.5}, \text { HOMA- } \beta=\frac{20 x \operatorname{Insulin}}{\text { Glucose }-3.5} \%, \quad H O M A-S=\frac{1}{\text { HOMA } \beta}
$$

Figure $1 \mathrm{HOMA}$ calculations where glucose is $\mathrm{mmol} / \mathrm{L}$ and insulin is $\mathrm{mU} / \mathrm{L} .{ }^{15} \mathrm{HOMA}$, homeostasis model assessment; HOMA- $\beta$; HOMA to assess pancreatic beta-cell function; HOMA-IR, HOMA to assess insulin resistance; HOMA-S, HOMA to assess insulin sensitivity. 
a small-amplitude high-frequency oscillation (periodicity $3-10 \mathrm{~min}) .{ }^{13}{ }^{14}$ It is hypothesised that the periodic release of insulin prevents downregulation of receptors leading to insulin resistance. Loss of these regular insulin oscillations is considered to be an early indicator of diabetes.

However, these oscillations make a single sample of insulin impractical for clinical practice, as it will be unknown where the insulin levels lie in these periodic cycles. It has been suggested that if fasting insulin concentrations are required, then the average of three samples, taken at 5 min intervals, should be used; however, this rarely is done in clinical practice. ${ }^{15}$

\section{Reproducibility of single fasting insulin measures}

Insulin resistance is extensively assessed in epidemiological studies, usually by measures that use fasting insulin. However, there are a number of questions about the reproducibility of fasting insulin. Coefficient of variation (CV) for fasting insulin has been reported between 25\% and $50 \%,{ }^{16}$ whereas other studies have reported CV of HOMA closer to $10 \% .^{15}$ Further work is needed on the reproducibility of measures using fasting insulin if these are to be used more in the clinical setting.

\section{INSULIN RESISTANCE}

There is a current argument as to whether we should be more concerned about insulin resistance or hyperinsulinaemia. As discussed by Crofts, ${ }^{4}$ insulin resistance is the state where the body does not respond to normal levels of insulin. The gold standard test for insulin resistance is the hyperinsulinaemia-euglycaemia clamp test, which assesses the glucose uptake rate. Tests such as HOMA have been validated against the clamp test. However, it is currently being argued that insulin resistance per se is not the major cause of physiological damage. People who are insulin-resistant become hyperinsulinaemic following a carbohydrate load, and as discussed above hyperinsulinaemia is also linked to adverse pathophysiological states. Since hyperinsulinaemia generally results from a postprandial state, ${ }^{9}$ it remains unknown whether fasting measures such as HOMA are sufficiently accurate to predict postprandial hyperinsulinaemia.

\section{RECOMMENDED ASSAY}

Although it is hyperinsulinaemia that is best assessed following an oral glucose load, there is debate as to the best test protocol. Currently, the two published tests are the Kraft and Hayashi protocols described above. The overarching principles for the two tests are the same.

The patient prepares by having at least 2 weeks of a diet containing at least $150 \mathrm{~g}$ carbohydrate per day. ${ }^{7}$ After an overnight fast ( $>10$ hours), the patient has a $75 \mathrm{~g}$ OGTT with plasma glucose and insulin sampled at baseline, 30, 60, 120 and $180 \mathrm{~min}$. (NB: if only the Hayashi protocol is being followed, then the $180 \mathrm{~min}$ sample may be omitted.) The results can be interpreted as per tables 1 and 2. It should be noted that the original Kraft

\begin{tabular}{|c|c|}
\hline Kraft pattern & Description \\
\hline $\begin{array}{l}\text { Pattern I } \\
\text { Normal insulin }\end{array}$ & $\begin{array}{l}\text { Fasting insulin } \leq 30 \mu \mathrm{U} / \mathrm{mL} \\
30 \text { min or } 1 \text {-hour peak } \\
\text { 2-hour+3-hour sum }<60 \mu \mathrm{U} / \mathrm{mL}\end{array}$ \\
\hline $\begin{array}{l}\text { Pattern IIA } \\
\text { Borderline }\end{array}$ & $\begin{array}{l}\text { Fasting insulin } \leq 50 \mu \mathrm{U} / \mathrm{mL} \\
30 \text { min or } 1 \text {-hour peak } \\
2 \text {-hour+3-hour sum } \geq 60,<100 \mu \mathrm{U} / \mathrm{mL} \\
\text { OR } \\
\text { Fasting insulin } 31-50 \mu \mathrm{U} / \mathrm{mL} \\
30 \text { min or } 1 \text {-hour peak } \\
2 \text {-hour+3-hour sum }<60 \mu \mathrm{U} / \mathrm{mL}\end{array}$ \\
\hline $\begin{array}{l}\text { Pattern IIB } \\
\text { Hyperinsulinaemia }\end{array}$ & $\begin{array}{l}\text { Fasting insulin } \leq 50 \mu \mathrm{U} / \mathrm{mL} \\
30 \text { min or } 1 \text {-hour peak } \\
\text { 2-hour+3-hour sum } \geq 100 \mu \mathrm{U} / \mathrm{mL}\end{array}$ \\
\hline $\begin{array}{l}\text { Pattern III } \\
\text { Hyperinsulinaemia }\end{array}$ & $\begin{array}{l}\text { Fasting insulin } \leq 50 \mu \mathrm{U} / \mathrm{mL} \\
\text { Delayed peak ( } 2 \text { hours or } 3 \text { hours) }\end{array}$ \\
\hline $\begin{array}{l}\text { Pattern IV } \\
\text { Hyperinsulinaemia }\end{array}$ & Fasting insulin $>50 \mu \mathrm{U} / \mathrm{mL}$ \\
\hline $\begin{array}{l}\text { Pattern V } \\
\text { Hypoinsulinaemia }\end{array}$ & All values $\leq 30 \mu \mathrm{U} / \mathrm{mL}$ \\
\hline
\end{tabular}

protocol was conducted with a $100 \mathrm{~g}$ oral glucose load. If a $75 \mathrm{~g}$ load is used, borderline results may also indicate hyperinsulinaemia. Further research is needed to confirm glucose dose and whether the protocol can be simplified.

Thus, a dynamic insulin assay combined with the glucose tolerance test that assesses insulin status following a glucose load is very likely to be worthwhile in adequately assessing the actual presence of diabetes, especially occult or diabetes in situ. Some limitations of the insulin assay include concomitant medications, varying assays, dietary factors (how long a person fasts), cultural and ethnic differences, and age of testing (puberty or postmenopausal for example). Thus, clearly more research is needed, but until then the insulin assay may be an improvement compared with an OGTT alone at diagnosing diabetes.

\begin{tabular}{ll}
\hline Table 2 Hayashi pattern results & \\
\hline Hayashi pattern & Description \\
\hline Pattern 1 & Peak at $30 \mathrm{~min}$ \\
Pattern 2 & Peak at $30 \mathrm{~min}$ \\
& $>60$ min $\leq 120 \mathrm{~min}$ \\
Pattern 3 & Peak at $60 \mathrm{~min}$ \\
Pattern 4 & Peak at $120 \mathrm{~min}$ \\
& $>30$ min $<60 \mathrm{~min}$ \\
Pattern 5 & Peak at $120 \mathrm{~min}$ \\
& $>30 \mathrm{~min} \geq 60 \mathrm{~min}$ \\
\hline
\end{tabular}

NB: If two equal peaks occur, then the earlier occurrence is deemed to be the peak. 


\section{CONCLUSION}

Diabetes is now a global pandemic, yet undiagnosed diabetes is a burgeoning and pernicious problem. The need to develop early biomarkers to diagnose diabetes is of utmost importance. The insulin assay, measuring both fasting and after an OGTT, seems to be the earliest biomarker for diagnosing T2D. There is a need to further test insulin assay algorithms and implement these diagnostic tools into the guidelines.

\section{Contributors All authors contributed to the final manuscript.}

Competing interests Dr. DiNicolantonio is the author of The Salt Fix and operates the website.

Provenance and peer review Not commissioned; internally peer reviewed.

Open Access This is an Open Access article distributed in accordance with the Creative Commons Attribution Non Commercial (CC BY-NC 4.0) license, which permits others to distribute, remix, adapt, build upon this work non-commercially, and license their derivative works on different terms, provided the original work is properly cited and the use is non-commercial. See: http://creativecommons.org/ licenses/by-nc/4.0/

(c) Article author(s) (or their employer(s) unless otherwise stated in the text of the article) 2017. All rights reserved. No commercial use is permitted unless otherwise expressly granted.

\section{REFERENCES}

1. CDC. News room press release. 2014 http://www.cdc.gov/media/ releases/2014/p0610-diabetes-report.html (accessed 10 Jun 2014).

2. Gregg EW, Zhuo X, Cheng YJ, et al. Trends in lifetime risk and years of life lost due to diabetes in the USA, 1985-2011: a modelling study. Lancet Diabetes Endocrinol 2014;2:867-74.
3. Menke A, Casagrande S, Geiss L, et al. Prevalence of and trends in diabetes among adults in the United States, 1988-2012. JAMA 2015;314:1021-9.

4. Crofts CAP. Hyperinsulinemia: a unifying theory of chronic disease? Diabesity 2015;1:34-43.

5. Kelly CT, Mansoor J, Dohm GL, et al. Hyperinsulinemic syndrome: the metabolic syndrome is broader than you think. Surgery 2014;156:405-11.

6. Kraft JR. Hyperinsulinemia: a merging history with idiopathic tinnitus, vertigo, and hearing loss. Int Tinnitus J 1998;4:127-30.

7. Kraft JR. Detection of diabetes mellitus in situ (occult diabetes). Lab Med 1975;6:10-22.

8. Kraft JR. IMx (Abbott) immunoassay of insulin: a practical alternative to ria hyperinsulinemia identification in idiopathic neurootology and other hyperinsulin metabolic disorders. Int Tinnitus J 1997;3:113-6.

9. Crofts C, Schofield G, Zinn C, et al. Identifying hyperinsulinaemia in the absence of impaired glucose tolerance: an examination of the kraft database. Diabetes Res Clin Pract 2016;118:50-7.

10. Hayashi T, Boyko EJ, Sato KK, et al. Patterns of insulin concentration during the OGTT predict the risk of type 2 diabetes in Japanese Americans. Diabetes Care 2013;36:1229-35.

11. Matthews DR, Hosker JP, Rudenski AS, et al. Homeostasis model assessment: insulin resistance and beta-cell function from fasting plasma glucose and insulin concentrations in man. Diabetologia 1985;28:412-9.

12. Levy JC, Matthews DR, Hermans MP. Correct Homeostasis Model Assessment (HOMA) evaluation uses the computer program. Diabetes Care 1998;21:2191-2.

13. Hellman B. Pulsatility of insulin release--a clinically important phenomenon. Ups J Med Sci 2009;114:193-205.

14. Schmitz O, Rungby J, Edge L, et al. On high-frequency insulin oscillations. Ageing Res Rev 2008;7:301-5.

15. Wallace TM, Levy JC, Matthews DR. Use and abuse of HOMA modeling. Diabetes Care 2004;27:1487-95.

16. Mather KJ, Hunt AE, Steinberg HO, et al. Repeatability characteristics of simple indices of insulin resistance: implications for research applications. J Clin Endocrinol Metab 2001;86:5457-64. 